\title{
eJRIEPS
}

Ejournal de la recherche sur l'intervention en éducation physique et sport

$23 \mid 2011$

Varia

\section{La technologie dans le contexte des STAPS : de la conception d'outils à la conceptualisation... et réciproquement}

\section{Gilles Uhlrich, Serge Eloi et Daniel Bouthier}

\section{(2) OpenEdition}

\section{Édition électronique}

URL : http://journals.openedition.org/ejrieps/4597

DOI : $10.4000 /$ ejrieps. 4597

ISSN : 2105-0821

Éditeur

ELLIADD

\section{Référence électronique}

Gilles Uhlrich, Serge Eloi et Daniel Bouthier, « La technologie dans le contexte des STAPS : de la conception d'outils à la conceptualisation... et réciproquement », eJRIEPS [En ligne], 23 | 2011, mis en ligne le 01 juillet 2011, consulté le 21 février 2020. URL : http://journals.openedition.org/ejrieps/4597 ; DOI : 10.4000/ejrieps.4597

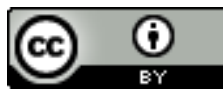

La revue eJRIEPS est mise à disposition selon les termes de la Creative Commons Attribution 4.0 International License. 
La technologie dans le contexte des STAPS : de la conception d'outils à la conceptualisation... et réciproquement

Gilles Uhlrich*, Serge Eloi** et Daniel Bouthier***

* Université Paris-Sud 11, UFR STAPS, UR CIAMS/ECAMS, Bâtiment 335, 91405 Orsay Cedex, France

** Université Paris-Est Créteil Val-de-Marne, Laboratoire REV/CIRCEF, 61 avenue du Général de Gaulle 94010, Créteil Cedex, France

*** IUFM d'Aquitaine, Avenue Léon Duguit, 33608 Pessac Cedex, France

Résumé de l'article

Lors de ce travail, les auteurs cherchent à resituer les recherches technologiques en STAPS. Ils proposent certains éléments permettant de les organiser selon les besoins de leurs utilisateurs. Ils distinguent d'abord une position théorique qui interroge les rapports entre technologie et sciences. L'originalité technologique est alors mise en exergue. Puis, ils décrivent une position plus pragmatique qui fait apparaître la technologie comme un projet d'intervention sur le réel en traquant les savoirs de l'agir. Cette caractérisation amène à mettre en rapport, dans une perspective de formation en sport collectif, conceptualisation en acte et action conceptualisée. Enfin, ils adoptent une position technicienne qui suggère un lien entre technique et technologie. Ils décrivent alors le rapport organisé entre technique, technicité et registres de technicité. Ainsi, les registres deviennent une catégorisation dynamique des recherches technologiques.

\section{Introduction}

Un certain nombre de travaux sur la technologie évoquant notamment son rapport à la science sont parus (Combarnous, 1982 ; Haudricourt, 1987 ; Staudenmaier, 1988). De nombreuses réflexions en termes d'objets et de démarches (Mouchet, Amans-Passaga \& Gréhaigne, 2010) semblent pouvoir alimenter le domaine de la recherche en Sciences et Techniques des Activités Physiques et Sportives (STAPS). Ceci apparaît d'autant plus important que les recherches technologiques à propos des sports collectifs dans leur double articulation «pratique du joueur » / «intervention éducative sur ce pratiquant » semblent être ignorées. II apparaît pourtant que cet aspect soit particulièrement sensible 


\section{eJRIEPS 23 avril 2011}

en formation initiale aux métiers de l'intervention éducative en sport. L'objectif de cet article est donc double :

- D'une part questionner les travaux de R. Deleplace (1979) puis d'A. Durey et D. Bouthier (Bouthier, 2008 ; Durey \& Bouthier; 1994) afin de reposer le cadre théorique des recherches technologiques.

- D'autre part, interroger la communauté des chercheurs en STAPS en situant leurs travaux par rapport au cadre de l'approche technologique et dans le même temps mettre en évidence un certain nombre de résultats de recherche pour la formation aux sports collectifs en STAPS.

Dans le cadre d'une articulation de la pratique avec la théorie, nous présentons ici quelques éléments de réflexion concernant le rapport entretenu entre science et technologie, éléments qui mettent en évidence la logique qui mène du pragmatique au conceptuel.

\section{1. Contexte de cette interrogation}

Nous souhaitons relancer la réflexion relative à l'enseignement des sports collectifs. En effet, alors que beaucoup d'intervenants déclarent envisager les sports collectifs dans une perspective dialectique (prise en compte systématique du rapport d'opposition), la réalité des pratiques d'enseignement et d'entraînement est tout autre (Brau Antony, 2001). La perspective techniciste (recours à une mobilisation étroite de savoir faire sans prise en compte du contexte) reste prépondérante. II est avéré depuis un certain temps qu'un décalage entre le « dire » et le « faire » existe. II en est ainsi d'enseignants d'EPS qui se déclarent plutôt «dialectiques » alors même que leurs propositions de dispositifs d'évaluation relèvent explicitement d'options particulièrement «technicistes » (Brau Antony, 1998). Cet écart interroge la formation initiale en STAPS. Comment peut-on créer les conditions d'un rapprochement des discours et des actes? La responsabilité des formateurs de formateurs se trouve donc engagée dans la propagation des méthodes les plus adéquates à l'enseignement des sports collectifs. De ce fait, il nous semble opportun de capitaliser le travail considérable fourni par Deleplace (1966, 1979, 1983, 1986, 1999). En effet, cet auteur a su développer une modélisation du rugby dont l'un des axes forts repose sur la prise en compte de l'adversaire. En se démarquant du modèle anglo-saxon techno centré au profit d'une formation qui épouse la complexité des activités physiques, il apparaît que Deleplace a ouvert la voie à d'autres travaux de modélisations dans d'autres sports collectifs. Cette relation dialectique « forme de l'attaque » / « adaptation défensive » questionne incontestablement la recherche en STAPS. Si nous considérons que les pratiques physiques et sportives doivent être au cœur de la recherche, il nous semble 


\section{eJRIEPS 23 avril 2011}

décisif de proposer des enseignements qui se nourrissent de ces travaux de recherches, et pas seulement en physiologie, psychologie ou anatomie. La controverse sur le poids des pratiques physiques et sportives lors de la formation n'est donc pas close. Ainsi, il paraît opportun de relancer le débat relatif au développement des méthodes d'enseignement. C'est dans cette logique que nous allons tenter d'éclairer quelques concepts.

\section{2. Des questions}

Notre réflexion porte sur la place et l'originalité de la recherche technologique en STAPS. Pour cela, nous avons tenté de répondre à plusieurs questions :

- Quel rapport existe-t-il entre science et technologie ? Une tentative de réponse à cette question ne peut pas être sans conséquence sur les conditions particulières d'une recherche technologique.

- Si la démarche technologique conduit à intervenir sur le réel en traquant les savoirs de l'agir, comment s'organiser pour prendre en compte cette double exigence ?

- Quelles sont les relations possibles entre les techniques et registres de technicité dans les activités physiques et sportives (APSA) ?

\section{Rapport technologie et science : position théorique}

Notre première question porte sur les rapports entre science et technologie. Nous allons évoquer ici quelques idées qui mettent en interaction ces deux concepts.

\section{1. Articulation science et technologie}

La théorie technologique vise à apprécier le pertinent, l'efficace, en déterminant des règles pour résoudre de façon optimale les problèmes de la pratique. Ces règles bouleversent les données sociotechniques (évolution de l'usage des techniques, apparition de nouveaux outils, transmission des techniques corporelles) dans tous les secteurs (pratique fédérale, Education Physique et Sportive (EPS), pratique de loisirs). Haudricourt (1987) la considère comme une science humaine des techniques. La recherche technologique évite la fragmentation entendue comme une morcellisation de l'objet de recherche. Elle conserve la complexité de la pratique comme une condition de la recherche. Ainsi, la recherche technologique se formalise au fur et à mesure du projet. Elle s'appuie plutôt sur des expériences contrôlées, en construisant des protocoles ancrés dans la pratique réelle ce qui engendre quelques pertes de données et de la prudence dans les conclusions. Pour Staudenmaier (1988), l'activité scientifique est souvent motivée par la curiosité. De son côté, la technologie est motivée par le désir de résoudre des problèmes. Dans ce contexte, si la science vise à construire des modèles théoriques, la technologie s'attache à 


\section{eJRIEPS 23 avril 2011}

produire des connaissances mises au service de cette construction. Par exemple, les travaux sur la formation aux prises de décision en sports collectifs, mettent en évidence un « double registre décisionnel mobilisé en jeu prédictif ou planifié, et réactif ou régulé » (Grehaigne, Godbout \& Bouthier, 2001). Mouchet montre, dans ce contexte, que les joueurs ont des prévalences individuelles pour l'un des deux registres selon les conceptions du jeu et les formations reçues (Mouchet \& Bouthier, 2006). Au-delà des sports collectifs, ces recherches technologiques interpellent les modèles théoriques, cognitivistes, écologiques et énactifs. Elles mettent en question l'idée scientifique qu'il existe une «transition entre fenêtres attentionnelles et émergences non intentionnelles avec des possibilités de passage ultra rapides et réversibles " comme modalité d'initialisation et de contrôle des actions sportives complexes (Bouthier, 2008, p. 56). En retour, les sciences inspirent la créativité technologique et rationalisent les pratiques existantes dans une perspective systémique. La technologie contribue donc à la science en produisant des instruments, en posant des questions scientifiques et en créant des nouveaux modèles conceptuels qui deviendront éventuellement scientifiques plus tard.

\section{2. L'originalité technologique dans le champ scientifique}

Pour Staudenmaier (1988), le savoir technologique est un style de connaissances propre et particulier intégrant des concepts scientifiques, des données spécifiques, des théories distinctes des sciences et du savoir faire. II faut comprendre par là que le savoir technologique emprunte aux champs scientifiques par l'utilisation de concepts (épistémologie, méthodes, méthodologies) mais aussi qu'il se nourrit de données incertaines isolées des pratiques et d'habiletés techniques en lien avec les motricités complexes de chacun.

Dans le domaine des STAPS, cette position permet d'associer à des concepts scientifiques clefs (mouvement comme conduite humaine) des données incertaines (incident $\mathrm{du}$ jeu) et des habiletés techniques (créations techniques). La technologie se nourrit des concepts scientifiques enchâssés autant dans la réalité de la pratique sportive que dans celle de l'intervention éducative en sport.

\section{3. La technologie dans le champ de l'intervention éducative}

Les recherches technologiques dans le cadre de l'intervention éducative, et plus particulièrement ici des sports collectifs, donnent du sens à l'efficience technique. Cette relation peut être schématisée comme suit (figure 1) : 


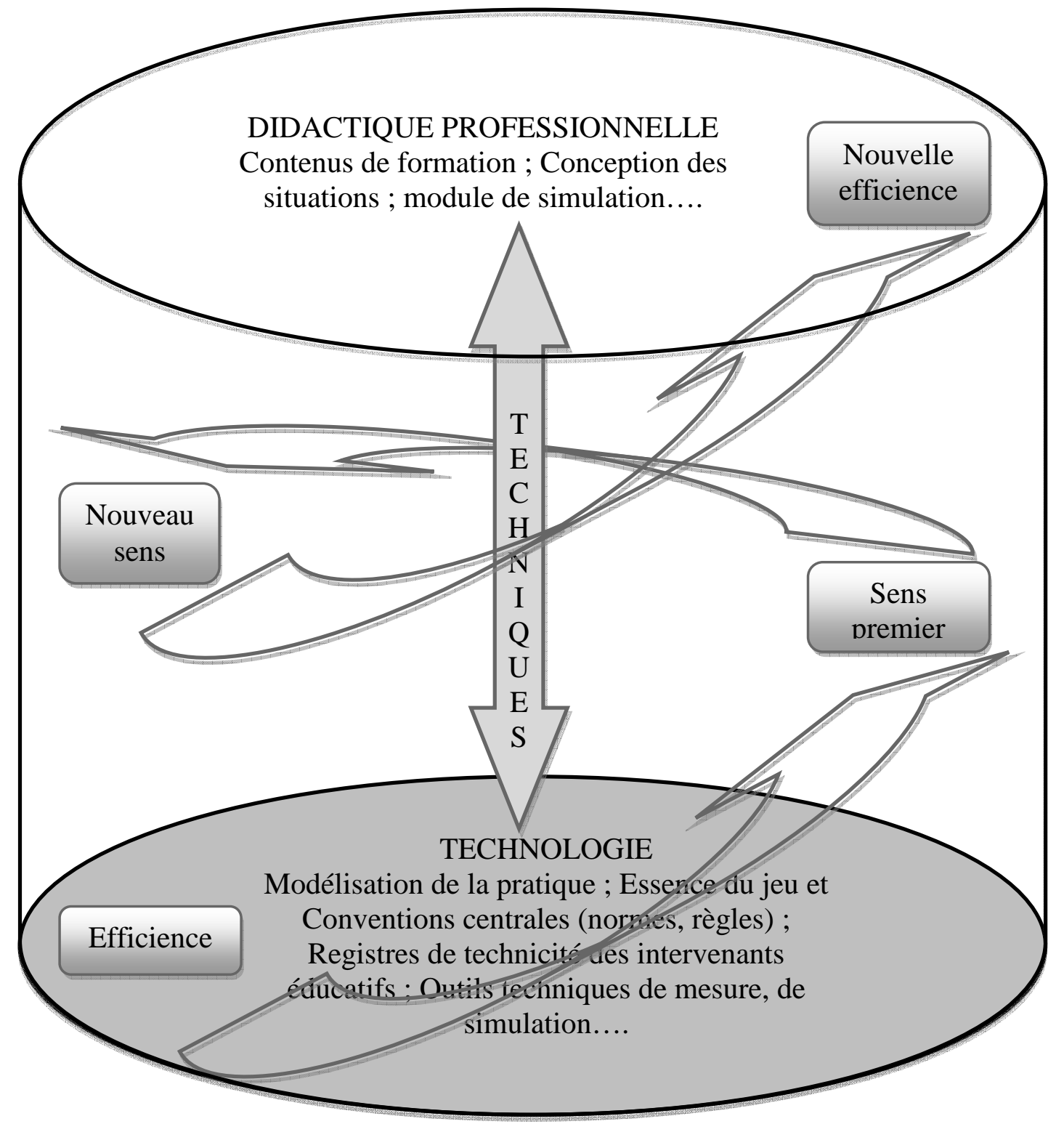

Figure 1. Perspective spiralaire de la symbiose Technologie/Didactique d'après Bouthier (2008).

Faisons fonctionner le schéma en développant rapidement un exemple de recherche. Considérons l'efficience du jeu de l'équipe Nationale des Fidji en rugby. Ces joueurs sont présentés dans la noosphère (la sphère des gens qui pense sur le rugby) comme des "créateurs" du fait qu'ils sont spécialistes du rugby à 7. Ils déstabiliseraient les adversaires parce que leur jeu est imprévisible. C'est leur particularité culturelle qui serait le sens premier de leur efficacité. Ainsi l'animation tactique de leur jeu expliquerait leur efficience (1/4 de finaliste de la coupe du monde 2007). Une recherche technologique portant sur le jeu des équipes nationales en rugby, comparant la coupe du monde 1999 et 


\section{eJRIEPS 23 avril 2011}

la coupe du monde 2007 nous a permis d'interroger cet a priori (Uhlrich, Mouchet, Bouthier, Fontayne, à paraître). Nous nous sommes appuyés sur la modélisation du jeu réalisée par R. Deleplace (1979) pour décrire les matchs. Ce dernier distingue trois plans d'analyse : le plan collectif total (équipe/équipe), le plan collectif de ligne $(7 / 7,8 / 8)$ et le plan Homme/Homme. Lors de ce travail, nous avons décrit le jeu des équipes au plan collectif total. Nous avons comparé ces enchaînements d'actions pour 8 équipes sur chacune des coupes du monde (1999 et 2007). Par ailleurs, nous avons tenté de repérer ce qui a évolué dans le jeu de chaque équipe d'une coupe du monde à l'autre. Pour ce faire nous avons utilisé un outil de description du jeu déjà éprouvé : le scénario de match (Mouchet, Uhlrich \& Bouthier, 2005). Durant ce travail, nous avons distingué d'une part, les «tendances de jeu communes lors d'une coupe du monde » et d'autre part, les « particularités de certaines équipes» (Mouchet, Uhlrich \& Bouthier, 2008). Les tendances communes correspondent à des formes de jeu utilisées par toutes les équipes. Les particularités représentent les singularités de certaines équipes dans ce contexte général. Les résultats de cette recherche montrent que l'efficacité des Fidjiens vient davantage d'une évolution significative de leur jeu entre deux coupes du monde (comparaison entre 1999 et 2007), plus que par l'accentuation d'une singularité de leur jeu. C'est par une adhésion aux tendances communes à toutes les équipes de la compétition (pourcentage de points de fixations, forme simple dans le mouvement général, etc.) que les Fidjiens ont évolués. Cette mutation s'explique par le fait que cette équipe est dirigée depuis plusieurs années par des entraineurs venus d'autres pays qui ont donc des cultures différentes (Nouvelle Zélande). La migration des joueurs est aussi devenue plus importante. Nombres de joueurs Fidjiens évoluent dans les championnats de rugby de l'hémisphère Nord (Angleterre, France). C'est donc davantage par un amalgame entre une culture Fidjienne faites d'initiatives et de prises de risques et de la maitrise d'un cadre plus «traditionnel » du jeu que cette équipe a progressé dans le concert international. Dans la reconstruction d'un sens nouveau sur l'efficience, nous montrons ici que c'est la capacité à faire évoluer le jeu d'une équipe en réalisant la cohésion entre singularité des joueurs et cadre général du jeu qui crée les conditions du progrès. Ce sens nouveau finalise la première boucle du schéma (efficience/sens premier/nouveau sens). Le jeu d'une équipe ne peut donc pas se réduire à une impression simpliste répandue de bouche à oreille à l'image d'une rumeur. Par l'analyse rigoureuse et technologique, nous dégageons d'autres conclusions. L'efficience d'une équipe repose sur des éléments plus complexes qui se retrouvent en interaction. Ce nouveau sens peut alors être réinvesti. Pour l'entraineur, il s'agira d'envisager à tout moment cette relation complexe : s'imprégner de la «culture » d'une 


\section{eJRIEPS 23 avril 2011}

équipe pour l'utiliser tout en essayant de lui adapter un cadre plus général de jeu considéré comme efficace. Ce résultat sert alors de point de départ à la boucle de retour de la figure 1 (nouveau sens/nouvelle efficience). II interroge les compétences professionnelles (aspects de didactique professionnelle) des futurs intervenants éducatifs en remettant en question l'idée de contenus de formations des futurs entraineurs strictement organisés autour d'a priori «culturels » et «techniques » ou du projet de jeu « magique ». Pour notre exemple, la nouvelle efficience consiste à faire en sorte que les futurs entraîneurs ou enseignants se positionnent dans une perspective d'identification, d'adaptation, voire d'innovation dans le jeu de leur équipe. Cette nouvelle efficience devrait être un objectif de préprofessionnalisation. On voit, à travers cet exemple que la relation «efficience/sens » des éléments techniques détermine l'orientation principale d'une recherche technologique du fait qu'elle interroge la réalité du jeu produit. Les éléments dégagés de la recherche peuvent alors être réinterrogés dans le sens inverse « sens/efficience » qui alimente alors plutôt une recherche en didactique professionnelle du fait que ce sont les compétences professionnelles qui doivent faire preuve de leur pertinence. Cette interaction n'est pourtant pas si dichotomique qu'elle peut y paraître :

- D'une part, parce que nombre de travaux associent démarche technologique et visée didactique (Eloi, 1997 ; Mouchet, 2004 ; Ulrich, 2005)

- D’autre part, parce que les connaissances dégagées par les travaux posent toujours de nouvelles questions. La connaissance n'est pas figée. C'est pour cette raison que le schéma présente des flèches qui montent dans un mouvement spiralaire. Nous avions précédemment évoqué cette idée de «spirale de complexification » (Uhlrich, Robert \& Pujos, 2000). Le concept développé ici postule qu'une entrée dans ce type de processus de formation provoque une évolution sans limite.

La figure 1 tente donc de rendre compte de cette dynamique sous la forme d'une spirale de complexification croissante.

\section{4. Conséquences}

Pour caractériser les conditions particulières d'une recherche technologique, nous pouvons alors proposer :

- La science et la technologie ont des rapports imbriqués. La technologie dont les situations typiques relèvent d'une démarche pragmatique n'occulte pas pour autant les concepts scientifiques qu'elle interroge au travers de l'évolution des techniques.

- La technologie prend en considération les problèmes de terrain. Ces obstacles constituent le point de départ de la démarche qui se concrétise en cours de recherche par un affinement et une évolution de ces outils (méthodologie, dispositif, artefacts...). 


\section{eJRIEPS 23 avril 2011}

- La technologie prend aussi en considération la complexité. La multiplicité des composants ou la diversité de leurs interrelations ne sont que la forme visible d'un système. Ce qui en fait la complexité, c'est l'imprévisibilité potentielle des conduites de ce système et le primat de l'action en contexte. «La notion de complexité implique celle d'imprévisible possible, d'émergence plausible du nouveau et du sens au sein du phénomène que l'on tient pour complexe »(Le Moigne, 1999, p.3). Aussi, se positionner dans une perspective de compréhension de l'efficience de la pratique c'est donner du sens aux éléments techniques. C'est de fait, alimenter une spirale de complexification croissante.

\section{Rapport technologie et pratique de terrain : position pragmatique}

Guillerme (1973) propose de définir la technologique comme l'usage rationnel des techniques dans le cadre de la conception, la mise en œuvre et la réalisation d'un projet d'intervention sur le réel. Bouthier quant à lui considère que la recherche technologique ne peut se faire qu'en traquant les savoirs de l'agir (Bouthier, 2005). Nous proposons de réunir ces deux idées pour n'en faire qu'une. En effet, il nous semble opportun de définir la position pragmatique comme un projet d'intervention sur le réel dont la méthode consiste à traquer les savoirs de l'agir. Tentons de saisir la portée des deux versants de cette position qui consiste à « conduire un projet d'intervention sur le réel pour traquer les savoir de l'agir ».

3. 1. «Conduire un projet d'intervention sur le réel... »

L'approche technologique réintroduit la technique au cœur des réflexions sur l'intervention éducative en sport. Cette réhabilitation de la technique (notion de technicité - voir $§ 4.1$ ) passe par des analyses et des modélisations de pratiques à des fins d'intervention et de transformation du jeu. Ainsi, ce type de recherche nécessite de se confronter à différents obstacles : recueils de données compliqués à «borner», cadre théorique adossé à diverses références épistémologiques, difficultés à généraliser les conclusions. Du fait de ces difficultés, cette démarche est trop souvent abandonnée. Dans cet ordre d'idée, on peut se poser la question de savoir si l'on doit encore s'interroger sur le référentiel commun d'action. En effet, il apparaît que la lecture du jeu au travers d'une logique de matrices (Deleplace, 1979) reste problématique à enseigner bien qu'elle constitue la possibilité pour les pratiquants d'anticiper sur la lecture du jeu des partenaires comme des adversaires. Notre réponse est affirmative car cette définition deleplacienne du référentiel commun offre alors une vision dynamique de la prise de décisions en jeu. Ce travail de modélisation des activités sportives nous engage à questionner certains travaux. En effet, 


\section{eJRIEPS 23 avril 2011}

il apparaît que le référentiel commun soit envisagé chez certains auteurs comme de simples «connaissances partagées» (Bourbousson \& Sève, 2010). On peut alors s'interroger sur la pertinence d'une telle distorsion vis à vis du concept développé initialement par Deleplace (Deleplace, 1979). Car si le référentiel commun est bien un système partagé et dynamique de lecture du jeu, il n'en demeure pas moins qu'il émane d'abord d'une modélisation de l'activité faite par un spécialiste. Ainsi, ce travail de modélisation sert de fil conducteur à la discussion que des élèves peuvent partager. II nous semble que c'est à cette condition que le « débat d'idées » (Grehaigne, 2007) peut avoir du sens. L'élève doit se saisir des avancées culturelles qui émanent de l'évolution historique des pratiques corporelles. II ne devrait pas avoir à réinventer le monde dans lequel il vit au risque de rester l'éternel débutant tant décrié. Tout le travail de l'enseignant consiste à transmettre les moyens qui permettent à l'élève de comprendre et de s'inscrire dans ce référentiel. C'est à cette condition nous semble-t-il qu'il sera en mesure de le mettre en œuvre dans la réalité du rapport d'opposition. Il y a donc nécessité de se référer à une modélisation du jeu qui permet d'orienter les prises de décisions inhérentes à la mobilisation de ce référentiel. Cette démarche réclame auparavant (avant l'acte d'enseignement) qu'une modélisation soit élaborée par des experts de la discipline sportive (enseignants, entraineurs, chercheurs, etc.). II se trouve que dans la plupart des cas, cette référence à une modélisation se fait de façon implicite, sans que l'intervenant n'en ait une connaissance précise. Ce qui peut expliquer un certain décalage entre le «faire » et le « dire». Mener un projet d'intervention sur le réel nécessite donc pour l'intervenant, de formuler la modélisation sur laquelle il s'appuie.

3. 2. «...pour traquer les savoirs de l'agir»

Dans ce contexte, la recherche technologique prend en compte la pratique sportive et l'intervention éducative en tant que complexités emboitées. II ne s'agit donc pas de se limiter à l'analyse de techniques isolées ou des dispositifs pour eux-mêmes. Nous analyserons ces emboitements en prenant en compte trois points de vue à partir desquels peuvent se construire des compétences. II s'agira donc de prendre en compte systématiquement, ces trois postures.

Le premier point de vue concerne le pratiquant : lorsqu'il est en position d'apprentissage ou d'entraînement, le pratiquant est plongé dans une situation particulière. II semble pertinent de montrer comment ce dispositif donne du sens (ou non) à son activité lui offrant alors la possibilité de générer une conceptualisation en action (Vergnaud, 1996). Par ailleurs, une telle situation devrait provoquer en retour, une réflexion sur son action lui permettant ainsi de saisir davantage que les règles de fonctionnement de la situation 


\section{eJRIEPS 23 avril 2011}

présente. II devrait pouvoir en comprendre des principes communs, généralisables et transposables. On pourra parler alors d’action conceptualisée.

Le deuxième point de vue concerne l'intervenant: acquérir le statut d'intervenant nécessite d'être capable de mener de conserve deux tâches indissociables. D'une part, mettre en place une situation dont on espère qu'elle poursuit un objectif explicitable. Ici, nous sommes au plan de l'action conceptualisée. D'autre part, être capable « in vivo », dans l'urgence de l'action éducative, de saisir toutes les opportunités qui se présentent pour adapter le dispositif initial en faisant évoluer la situation, en invitant le pratiquant à s'engager délibérément dans une voie de réalisation. Le but est alors de montrer au pratiquant que l'action spécifique qu'il vient de réaliser est caractérisable, généralisable et transposable. Dans ce cadre nous faisons référence à la conceptualisation en acte.

Le troisième point de vue s'intéresse à la formation du futur intervenant : en prenant en compte les deux premiers points de vue, il apparaît que celui de la formation des intervenants ne peut être négligé. On ne peut pas envisager cette formation sans prendre en compte la double exigence suivante : en premier lieu, l'intervenant doit avoir vécu les classes de situations qu'il est amené à proposer et à faire vivre. En second lieu, il doit être formé à l'analyse de ces situations dans la perspective de faire expérimenter à ses pratiquants le double emboitement de l'action conceptualisée et de la conceptualisation en acte. Se pose alors le problème pour les formateurs de formateurs d'enseigner aux futurs intervenants à gérer dans le même temps, la régulation pédagogique de la situation, l'analyse des indices qui engage à adapter le dispositif initial et la possibilité de saisir les opportunités de systématisation des actions développées par les pratiquants pour montrer leur capacité à être caractérisable, généralisable et transposable. Face à un tel défi, il semble bien qu'une solution possible passe par l'utilisation et le contrôle de différents artefacts. Ce point est développé dans l'article d'Eloi \& Ulrich dans ce numéro. Traquer les savoirs de l'agir peut donc prendre diverses formes selon les points de vues envisagés ou l'angle d'attaque des phénomènes de conceptualisation.

\section{Rapport technologie et technique : position technicienne}

Nous proposons maintenant de caractériser la technique dans la démarche technologique et d'envisager les conséquences de cette proposition en termes de concepts utiles pour les formations en STAPS.

\section{1. Rapport « technique et registre de technicité » sur les APSA}

Les APSA sont imprégnées de techniques. Ce constat est souvent mal interprété. Durant de nombreuses années, l'amalgame a été réalisé entre technique et gestuelle «idéale » 


\section{eJRIEPS 23 avril 2011}

(belle passe, belle réception, beau geste). Cet a priori devrait être dépassé. «Dans chaque domaine, la technique est l'ensemble des procédés qui se lèguent de génération en génération, se diffusent par la transmission orale, par l'apprentissage, par l'enseignement, et dont l'utilisation assure l'efficacité de l'action » (Combarnous, 1984, p.31). La mobilisation adéquate d'une technique fait alors appel à de la technicité qui s'exprime dans un rapport dialectique « objet de l'action/action ». Pour Combarnous, « trois composants premiers doivent pouvoir caractériser la technicité :

- Un composant d'apparence philosophique, la rationalité technique.

- Un composant matériel, l'emploi d'engins (outils, instruments, machines, équipements).

- Un composant sociologique, combinaisons des spécialisations et des organisations.

La réunion de ces composants, réunion qui se comporte comme un système, constitue la base de toutes les activités techniques »(Combarnous, 1982, p. 228). Dans le domaine des STAPS, et plus particulièrement en sports collectifs, la rationalité technique peut s'envisager sous l'aspect de problématiques, de concepts et de normes. Ce qui renvoie bien à des réflexions philosophiques. Une problématique possible concerne la prise en compte du rapport d'opposition. Les concepts développés peuvent avoir trait aux matrices (Deleplace, 1979), aux configurations (Grehaigne, 1992), aux situations de fixation (Éloi, 2009). Les normes quant à elles renvoient à une position éthique, à des connaissances règlementaires ou à des standards de production de jeu permettant l'évaluation et la hiérarchisation des joueurs. La composante matérielle qui s'exprime au travers de l'emploi d'outils et d'instruments matériels ou symboliques peut s'apparenter à des dispositifs de formation, à des situations pédagogiques, à des représentations de choix tactiques (arbres de décisions). Pour ce qui relève de l'élément sociologique et des concepts de combinaisons et spécialisation qui lui sont associés, les sports collectifs ne sont pas avares d'exemples. La spécialisation historique des joueurs aux différents postes montre que le chemin de l'efficacité a emprunté la voie de la détermination de profils particuliers. De la même manière, l'organisation des relations entre les joueurs aboutit souvent à la mise en place de schémas stratégico-tactiques pré-déterminés.

L'analyse des ces combinaisons de jeu en dit d'ailleurs beaucoup sur l'évolution du rapport d'opposition au fil du temps. C'est donc l'ensemble de ces composants qui peut expliquer le passage d'une conception de la technique comme un « objet figé » à une conception novatrice de la technique comme une capacité à pouvoir la mobiliser dans son milieu d'expression. Ainsi, le pratiquant intègre des techniques qui le conduisent par l'expérience sensible à un certain degré de technicité. De son côté, l'intervenant éducatif, après avoir mobilisé ces techniques, doit percevoir les technicités potentielles (capacité à 


\section{eJRIEPS 23 avril 2011}

utiliser les techniques) de ses pratiquants. En conséquence, ce rapport aux techniques interroge la formation en STAPS et particulièrement les enseignements sur les pratiques d'APSA. Pour envisager la technicité, le formateur de formateur doit alors dégager des problématiques, des concepts et des normes, des outils symboliques et matériels ainsi que la perception des conditions d'évolution de la technique. Pour décrire ces composantes de la technicité, Martinand (1994) parle de registres de technicités.

4. 2. Catégorisation des recherches technologiques: les registres de technicité pour les STAPS

Nous proposons une approche novatrice des techniques qui articule la prise en compte de l'individu dans la spécificité de sa motricité d'une part et l'intervention éducative de l'enseignant ou de l'entraîneur d'autre part (Clot, 1997 ; Durey 1997 ; Bouthier, 1997). Dans cette perspective, les recherches en technologie pourraient être caractérisées selon différents registres.

4. 2. 1. Les registres de technicités adaptés aux spécificités des APSA

Nous pouvons distinguer quatre registres de technicités pour caractériser les recherches technologiques sur les APSA (Martinand, 1994 ; Durey \& Bouthier, 1994). Bien que depuis la proposition initiale de Martinand (1994) le concept de registre a évolué, nous proposons de le définir ainsi :

- Le registre de maîtrise : il prend en compte les états de maîtrise des techniques. II correspond en premier lieu, aux habiletés sportives dans la logique du pratiquant, ensuite, aux techniques d'intervention dans la logique de l'intervenant et enfin, aux modalités de formation pour le formateur de formateurs.

- Le registre de lecture : selon les points de vue, ce registre recouvre les modalités de prises d'information sur les sujets qui sont le centre d'intérêt de la pratique. Ainsi, pour le pratiquant, c'est l'adversaire qui est (devrait être) le centre d'intérêt. Pour l'intervenant, c'est le pratiquant qui est l'objet de son attention. Enfin, pour le formateur de formateurs, ce sont les informations provenant du futur intervenant qui sont prioritaires.

- Le registre de transformation : il désigne les conditions et procédures d'évolution des savoirs techniques. L'appropriation et le développement de la technique à l'aune de sa propre sensibilité sont décisifs pour le pratiquant. L'adaptation des procédures de transmission des savoirs est une préoccupation constante pour l'intervenant. La mise en place des dispositifs d'aide à la construction des connaissances utiles à la formation des pratiquants est une des missions essentielles du formateur de formateurs.

- Le registre de participation: il relève de l'activité déployée en dehors de la pratique principale de la discipline sportive. Ceci vaut lorsque le pratiquant endosse les rôles 


\section{eJRIEPS 23 avril 2011}

d'arbitre, de manager, d'observateur outillé. II en est de même pour l'intervenant éducatif lorsqu'il revêt le costume de joueur, d'arbitre, d'organisateur de tournois, d'accompagnateur. Par ailleurs, on peut voir le formateur de formateurs adopter le statut de chercheur, de préparateur aux concours ou d'organisateur d'événements (séminaires, tournois, tournées).

Pour compléter le registre de participation il faut ajouter la possibilité, pour des personnes extérieures au processus de formations, d'intégrer la noosphère de l'activité en prenant le rôle d'arbitre, de dirigeant, de kinésithérapeute, de journaliste...

4. 2. 2. Les registres de technicité comme catégorisation dynamique des recherches technologiques

II nous semble avantageux de pouvoir caractériser les recherches technologiques par une dominante. Ainsi, le registre de lecture est prépondérant dans le cadre de la description du jeu en rugby (Ulrich \& Bouthier, 2008). C'est aussi le cas pour le registre de maîtrise à propos des passeurs de haut niveau en volley-ball (Eloi, 2009). Lors d'une recherche technologique sur les APSA, la prise en compte d'un registre principal amène à envisager les influences éventuelles des autres registres. S'il n'y a pas de hiérarchie dans les registres (Martinand, 1994) rien ne laisse supposer qu'ils soient isolés les uns des autres. C'est la raison pour laquelle nous proposons de distinguer « registre principal » et « registre(s) associé(s)». L'objectif est de mettre en évidence que l'approche technologique permet d'envisager, au-delà d'une composante initiale (notion de registre principal) les influences d'autres domaines (registres associés). Cette mise en relation permet d'anticiper sur les retombées possibles que peuvent produire les connaissances nouvellement dégagées. Et c'est bien là le propre des recherches technologiques. Ces connaissances ne restent pas sans effets sur les autres registres (registres associés). Ainsi, les avancées relatives au registre de lecture influencent les éléments constitutifs du registre de transformation. De fait, les outils d'observation du jeu amènent à envisager différemment la pratique. De la même façon, les résultats issus du registre de lecture peuvent avoir des conséquences auprès des législateurs des lois du jeu, conséquences qui s'inscrivent alors dans un registre de participation. La connaissance du véritable impact du libéro sur l'attaque en volley-ball relativise l'objectif des législateurs de renforcer le pouvoir de la défense (cf. Éloi dans ce numéro). Ainsi, les connaissances techniques issues de ces recherches technologiques contribuent à développer la culture relative aux pratiques étudiées. Cependant, si les résultats de ces recherches peuvent être exploités dans le cadre des enseignements de type technologique auprès des futurs intervenants, il reste un long chemin à parcourir pour les intégrer dans les dispositifs de formation. C'est à 


\section{eJRIEPS 23 avril 2011}

cette étape qu'il faut considérer un autre niveau d'exploitation de ces travaux. II est essentiel d'envisager les conditions d'appropriation de cette nouvelle technicité dans le cadre de l'intervention éducative et ce, pour tous les points de vues (pratiquants, intervenants éducatifs, formateurs de formateurs). Dans ce cadre, il est utile de mettre à contribution différents outils. Dans la mesure où ces outils peuvent revêtir la forme d'instruments, de dispositifs, de concepts, il nous semble judicieux de les nommer «artefacts » (Norman, 1993 ; Rabardel, 1995 ; Vérillon, 2005). Nous avons développé depuis plusieurs années des travaux à propos de l'utilité éventuelle d'artefacts lors du processus de formation (Uhlrich, 2005 ; Uhlrich, 2007 ; Uhlrich et Bouthier, 2008). II nous semble qu'une prise en compte de ces outils et de leurs relations avec les registres de technicité pourraient être un prolongement enrichissant de l'approche technologique pour les formations en STAPS.

\section{Bibliographie}

Bourbousson, J., \& Sève, C. (2010). Construction/déconstruction du référentiel commun d'une équipe de basket-ball au cours d'un match. eJRIEPS, 20. 5-25.

Bouthier, D. (1997). L'EPS et son rapport aux techniques. Spécial didactique ; L'EPS dans l'école et la société d'aujourd'hui. Spirales, 8, 96-98.

Bouthier, D. (2005). Analyse de l'Activité en APS et développement des Compétences en Didactique professionnelle. Séminaire ISA-IDEES.

Bouthier, D. (2008). Technologie des APSA : évolution des recherches et de leur place dans le cursus STAPS. eJRIEPS, 15, 44-59.

Bouthier, D., \& Durey, A. (1994). Technologie des APS. Impulsions, 1, 95-124.

Brau Antony, S. (1998). L'évaluation des jeux sportifs collectifs. Des conceptions desenseignants d'EPS à la démarche de mise en œuvre du projet d'enseignement/évaluation. Thèse de l'Université Paris XI, Orsay.

Brau Antony, S. (2001). Les conceptions des enseignants d'éduction physique et sportive sur l'enseignement et l'évaluation des jeux sportifs collectifs: résultats d'une enquête. STAPS, 56, 93-108.

Clot, Y. (1997). Technique et travail humain. Spécial didactique ; L’EPS dans l'école et la société d'aujourd'hui. Spirales 8, 90-93.

Combarnous, M. (1982). La culture face à la technique. Culture Technique, 7, 227-241.

Deleplace, R. (1966). Le Rugby. Analyse technique et pédagogique. Paris : Armand Colin. Deleplace, R. (1979). Rugby de mouvement, rugby total. Paris, Revue EPS. 


\section{eJRIEPS 23 avril 2011}

Deleplace, R. (1983). La recherche sur la spécialisation sportive, l'entraînement, la performance. Intervention au $2^{\text {ème }}$ colloque des UEREPS, Université de Nice, 93151.

Deleplace, R. (1986). La «modélisation » en sport collectif. Actes du colloque EPS: contenus et didactique. SNEP. 105-108.

Deleplace, R. (1999). 1945-1995 : l'EPS face au sport, 15 acteurs témoignent. Centre EPS et Société. 212 p.

Durey, A. (1997). L'EPS et son rapport à la technique, Spirales 8, 93-95.

Eloi, S. (2001). Représentations mentales et acquisition d'habiletés tactiques en volleyball, effets comparés de deux cycles d'enseignement. Science et motricité, 40, 2231.

Eloi, S. (2009). Style d'un passeur de haut niveau en volley-ball. eJRIEPS, 17, 76-105.

Eloi, S., \& Uhlrich, G. (2001). Contribution à la caractérisation des sports collectifs : les exemples du volley ball et du rugby. STAPS, 56, 109-127.

Guillerme, J. (1973). Technologie, Encyclopædia Universalis, 15.

Grehaigne, J.F. (1992). L'organisation du jeu en football. Paris : Actio.

Grehaigne, J.F. (2007). Configurations du jeu - Débat d'idées \& apprentissage du football et des sports collectifs. Besançon : PU de Franche Comté.

Grehaigne, J-F., Godbout, P., \& Bouthier, D. (2001). The teaching and learning of decision making in team sports. Quest, 53, 59-76.

Haudricourt, G. A. (1987). Technologie, science humaine. Paris : Maison des Sciences de l'Homme.

Le Moigne, J.L. (1999) La modélisation des systèmes complexes. Paris : Dunod (2e ed).

Martinand, J.L. (1994). La didactique des sciences et de la technologie et la formation des enseignants. ASTER, 19, 61-75.

Mouchet, A., \& Bouthier, D. (2006). Prendre en compte la subjectivité des joueurs de rugby pour optimiser l'intervention. STAPS, 72, 93-103.

Mouchet, A., Uhlrich, G., \& Bouthier, D. (2005). La continuité en rugby: théorie et méthodologie d'une étude comparative entre nations. Impulsion, 4, 81-101.

Mouchet, A., Uhlrich, G., \& Bouthier, D. (2008). Dynamique de production du jeu de mouvement, tendances communes et particularités au niveau international.

Mouchet, A., Amans-Passaga, C., \& Gréhaigne, J.F. (2010). L’approche technologique. In M. Musard, M. Loquet \& G. Carlier, Sciences de l'intervention en EPS et en sport. Résultats de recherches et fondements théoriques, (pp. 201-222). Paris: Ed. revue EP\&S. 


\section{eJRIEPS 23 avril 2011}

Norman D. A. (1993). Les artefacts cognitifs. Raisons Pratiques, 4, $15-34$.

Rabardel, P. (1995). Les hommes et les technologies. Approche cognitive des instruments contemporains. Paris : Armand Colin.

Staudenmaier, J. (1988). L'histoire des sciences et la question : « les technologies sontelles des sciences appliquées ? ». Courrier du Cethès, construire un éthique de l'enseignement scientifique, 5, 27-43.

Uhlrich G. (2005). Rôle des artefacts matériels et cognitifs dans le développement des compétences de l'intervenant éducatif et rugby. Thèse non publiée. Université Bordeaux 2, V. Segalen.

Uhlrich, G. (2007). Former à l'observation par la simulation. Former les enseignants d'EPS. SNEP, Contre-pied, 20, 49-51.

Uhlrich, G., Pujos, X., \& Robert, J. (2000). Le rugby. Actes du colloque EPS, quelle activité pour quelle réussite de tous ? Orsay, Mars 2000, SNEP. 65-72.

Uhlrich, G., Bouthier, D. (2008). Efficacité d'une situation de formation, par la simulation, à l'observation du mouvement général en rugby, eJRIEPS, 15,198-215.

Uhlrich, G., Mouchet, A., Bouthier, A., \& Fontayne, P. (à paraître). Genre et styles de jeu en rugby : comparaison de la coupe du monde 1999 et 2007.Revue @ctivités.

Vergnaud, G. (1996). Au fond de l'action, la conceptualisation. In J.-M. Barbier (Ed.), Savoirs théoriques et savoirs d'action, (pp. 275-292). Paris : PUF.

Vérillon, P. (2005). Processus productifs et constructifs dans les activités physiques et sportives : la place de l'instrument. Impulsions, 4, 305-325. 